\title{
BMJ Open Resource use and costs associated with epilepsy in the Queensland hospital system: protocol for a population-based data linkage study
}

\author{
Ruth Tulleners (D) , ${ }^{1}$ Robin Blythe (D) , ${ }^{1}$ Sasha Dionisio, ${ }^{2}$ Hannah Carter (D) ${ }^{1}$
}

To cite: Tulleners $R$, Blythe $R$, Dionisio $\mathrm{S}$, et al. Resource use and costs associated with epilepsy in the Queensland hospital system: protocol for a population-based data linkage study. BMJ Open 2021;11:e050070. doi:10.1136/ bmjopen-2021-050070

- Prepublication history for this paper is available online. To view these files, please visit the journal online (http://dx.doi. org/10.1136/bmjopen-2021050070).

Received 10 February 2021 Accepted 09 November 2021

A) Check for updates

C Author(s) (or their employer(s)) 2021. Re-use permitted under CC BY-NC. No commercial re-use. See rights and permissions. Published by BMJ.

${ }^{1}$ Australian Centre for Health Services Innovation and Centre for Healthcare Transformation, School of Public Health and Social Work, Faculty of Health, Queensland University of Technology, Brisbane, Queensland, Australia

${ }^{2}$ The University of Queensland, Saint Lucia, Queensland, Australia

Correspondence to Mrs Ruth Tulleners; ruth.tulleners@qut.edu.au

\section{ABSTRACT}

Introduction Epilepsy places a large burden on health systems, with hospitalisations for seizures alone occurring more frequently than those related to diabetes. However, the cost of epilepsy to the Australian health system is not well understood. The primary aim of this study is to quantify the health service use and cost of epilepsy in Queensland, Australia. Secondary aims are to identify differences in health service use and cost across population and disease subgroups, and to explore the associations between health service use and common comorbidities.

Methods and analysis This project will use data linkage to identify the health service utilisation and costs associated with epilepsy. A base cohort of patients will be identified from the Queensland Hospital Admitted Patient Data Collection. We will select all patients admitted between 2014 and 2018 with a diagnosis classification related to epilepsy. Two comparison cohorts will also be identified. Retrospective hospital admissions data will be linked with emergency department presentations, clinical costing data, specialist outpatient and allied health occasions of service data and mortality data. The level of health service use in Queensland, and costs associated with this, will be quantified using descriptive statistics. Difference in health service costs between groups will be explored using logistic regression. Linear regression will be used to model the associations of interest. The analysis will adjust for confounders including age, sex, comorbidities, indigenous status, and remoteness.

Ethics and dissemination Ethical approval has been obtained through the QUT University Human Research Ethics Committee (1900000333). Permission to waive consent has been granted under the Public Health Act 2005, with approval provided by all relevant data custodians. Findings of the proposed research will be communicated through presentations at national and international conferences, presentations to key stakeholders and decision-makers, and publications in international peer-reviewed journals.

\section{INTRODUCTION}

Epilepsy is a chronic neurological disorder characterised by recurrent unprovoked seizures with serious effects on neurobiological, cognitive, psychological and social

\section{Strengths and limitations of this study}

- The broad range of health service use and cost data linked from a variety of sources will allow for comprehensive and detailed results that can be disaggregated to population groups, syndromes and severities.

- The data collected will provide an evidence base to inform future cost-effectiveness studies of epilepsy treatments.

- The use of hospital admissions data to identify the cohort of epilepsy patients means that patients who have not presented to hospital over the data collection period will be excluded. The analysis will, therefore, be focused on patients whose epilepsy is not well controlled, or those who have comorbidities that result in hospital presentation.

- The linked dataset will not include health service use or costs incurred outside the hospital system, including costs associated with pharmaceuticals or primary care.

functioning. ${ }^{1}$ Globally, epilepsy affects $1 \%-2 \%$ of the population making it the most common neurological disorder ${ }^{2}$ with a burden of disease equivalent to lung cancer in men and breast cancer in women. ${ }^{3}$ It is commonly associated with mental health comorbidities, with around half of adults with epilepsy experiencing depression, ${ }^{4}$ a higher proportion than in any other chronic condition. ${ }^{5}$ In Australia, it is estimated that there are more than 250000 people currently living with epilepsy. ${ }^{6}$

Epilepsy places a large burden of health systems, with hospitalisations for seizures alone occurring more frequently than those related to diabetes. ${ }^{7}$ However, the cost of epilepsy to the Australian health system is not well understood. There is also a lack of evidence around how health service utilisation and costs vary among population groups, syndromes and severities. ${ }^{8}$ 
There is a need for improved access to health services for patients with epilepsy in Australia and globally. While pharmacological advances have enabled many patients to control their epilepsy through antiepileptic drugs (AEDs), approximately one-third of patients will be refractory to medications. ${ }^{9}$ While surgical intervention may be effective for many of these patients, epilepsy surgery remains heavily underused..$^{10}{ }^{11} \mathrm{~A}$ number of factors have been identified as contributing to this treatment gap, including knowledge gaps among physicians in identifying suitable candidates for surgery, lack of coordinated patient care, socioeconomic disparities that impede access to care, and the cost and complexities involved in the preoperative evaluation. ${ }^{12}$ As a result, patients with drug-resistant epilepsy are likely to experience recurrent healthcare needs and higher associated costs. This cohort has been estimated to account for $80 \%$ of direct and indirect costs of illness in epilepsy. ${ }^{13}$

Detailed information on the health service use and costs associated with epilepsy is required to inform costeffectiveness analyses of epilepsy treatments, where many knowledge gaps exist. These analyses provide decision makers with the evidence required to maximise health benefits from scarce resources. Outcomes on the differences in health service use in patient subgroups will also provide important insights into how the health and economic burden of epilepsy is distributed across society. This may inform policy decisions that direct resources to areas of greatest need.

Through this research, we aim to quantify the hospital and outpatient health service use and costs of patients with epilepsy; compare differences in health service use across patient subgroups including indigenous patients, children, rural/remote patients, and socioeconomically disadvantaged patients; and explore the associations between health service use and common comorbidities. This will be the first population-based analysis of the health service use and cost associated with epilepsy in the Queensland hospital system. It will build on the evidence produced in a 2018 study that was conducted in the neighbouring Australian state of New South Wales. However, key differences in the population characteristics of Queensland mean that this study may be able to provide a more comprehensive understanding of the nature and magnitude of health service use in Australia's Aboriginal and Torres Strait Islander peoples, and people living in regional and remote areas, where both groups are relatively highly represented. ${ }^{14}$ The use of comparison cohorts, as well as the planned regression analyses to investigate factors associated with resource use and cost, represent further contributions to the literature in this field. The evidence produced in this study may be used to inform healthcare priority setting and funding allocation. It may also provide information to support the design and evaluation of health service models of care and interventions for the identification and management of epilepsy.

\section{METHODS AND ANALYSIS}

\section{Setting and participants}

Queensland has a population of approximately 5 million people, with $36 \%$ of the population living outside of major cities, including $13 \%$ in outer regional areas, and $2 \%$ in remote or very remote areas. ${ }^{15}$ The state also has a relatively high proportion of Aboriginal and/or Torres Strait Islander peoples. It is known that these population groups have lower levels of access to health services than the general population, especially those living in metropolitan areas. ${ }^{16}$ These data will, therefore, provide an opportunity to explore differences in health service use and costs in under-represented populations.

A data linkage study will be undertaken via the Statistical Services Branch at Queensland Health. The base cohort will include all Queensland patients admitted to hospital with a principal or secondary diagnosis of epilepsy between 1 January 2014 and 31 December 2018 or later, depending on availability of updated datasets at the time of analysis.

Patients in the base cohort will be identified using International Classification of Diseases-10th Revision (ICD-10) diagnosis codes. ICD codes are applied internationally as a tool for collecting and analysing national epidemiological data. In Australia, they are routinely collected on presentation to emergency departments (EDs) and on admission to hospital. Current epidemiological guidelines suggest that a probable diagnosis of epilepsy can be made if one of the following three conditions is met: one medical encounter with a 3-digit code of G40.x (epilepsy); $\geq 2$ medical encounters on separate days coded with G41 (status epilepticus) or with a 4-digit code R56.8 (other and unspecified convulsions); and a single medical encounter coded as other and unspecified convulsions (R56.8) and an AED prescription for three or more months. ${ }^{17}$ However, coding processes are prone to human error and it has been recommended that their sensitivity and specificity is validated in specific populations and settings. An Australian study validating epilepsy diagnoses against routinely collected health data found that the highest positive predictive value $(81.4 \%)$ came from combining ICD-10 diagnosis codes and AED prescription data. ${ }^{18}$ In the absence of prescription data, the use of ICD-10 diagnosis codes of G40 and G41 produced the highest positive predictive value $(71.3 \%)$. Negative predictive values were highly precise $(99.9 \%)$. Based on these findings, we will include all patients with at least one admission that has been assigned an ICD-10 code of G40 or G41 in the base cohort. A subgroup analysis will adopt the more stringent criteria of requiring at least two such admissions.

ICD-10 codes will further allow for diagnoses to be disaggregated to the type of epilepsy, including whether the epilepsy is controlled or refractory, generalised or localised, with a known or unknown cause, and with or without the presence of specific epilepsy syndromes. The Charlson Comorbidity Index will be used to identify comorbidities using ICD-10 diagnosis codes from hospital records. Other common comorbidities in the cohort of 
people with epilepsy will also be identified from ICD-10 diagnosis codes and investigated within the analysis.

\section{Cohort of interest}

Two comparison cohorts will also be identified from the same time period for comparison purposes: (1) A diseaserelated cohort of patients admitted with any diagnosis of a nervous system disease (ICD-10 codes G00-G99); (2) A comparison cohort to be selected by randomly matching each index admission with on age, sex, indigenous status and Accessibility and Remoteness Index of Australia.

\section{Patient and public involvement}

Patients and the public were not involved in the research question, design, conduct, or choice of outcome measures for the study. The authors have, however, engaged with peak Australian and Queensland epilepsy advocacy bodies to assist in engaging with the public and patients during dissemination activities, and to determine ongoing research activities arising from the results of data analysis.

\section{Datasets and variables}

Retrospective hospital admissions data will provide detailed demographic data on each individual in addition to clinical information. Each individual identified in the base and comparison cohorts will then be linked across a number of other administrative datasets to obtain information on hospital admission costs, ED presentations, specialist outpatient and allied health occasions of service, mortality and cause of death. A list of the data collections to be linked is provided in table 1 .

Data variables from all data collections will be sourced and linked by the Statistical Services Branch, and sent to the researchers as a single file containing deidentified individually linked data. Figure 1 outlines the data linkage process.

\section{Research questions}

Specific research questions to be addressed using the linked dataset include:

- What level and type of health services are used by people with epilepsy, and what are the costs associated with these services?

- How does health service use differ between subgroups, including children, older people, indigenous and patients in rural/remote communities?

- What are the associations between epilepsy and common comorbidities, including mental health conditions and cognitive impairment?

- How does the presence of comorbidities impact on overall health service use and cost?

- What were the leading causes of death in the cohort?

- What proportion of ED presentations for seizure translated to an admission?

-What were the hospital outcomes for specific epilepsy syndromes, including status epilepticus (including length of stay and 30 day mortality rates)?

- What was the incidence of trauma related events in this cohort (including motor vehicle accidents and burns)?

\section{Statistical analysis}

All data will be analysed using the statistical software package R. An initial data cleaning process will be conducted to separately examine the distribution around each variable, address any implausible data points and correct for any structural issues across the datasets, for example, inconsistent naming conventions.

\begin{tabular}{|c|c|c|}
\hline Data collection & Description & Time period \\
\hline $\begin{array}{l}\text { Queensland Hospital Admitted Patient Data } \\
\text { Collection (QHAPDC) }\end{array}$ & $\begin{array}{l}\text { Collects demographic data and clinical information on } \\
\text { all admitted patients separated from both public and } \\
\text { licensed private hospitals and private day surgeries in } \\
\text { Queensland. }\end{array}$ & 1 January 2014 to 31 December 2018 \\
\hline $\begin{array}{l}\text { Emergency Department Information System } \\
\text { (EDIS) }\end{array}$ & $\begin{array}{l}\text { Provides data on care and treatment given to patients } \\
\text { in Queensland emergency departments }\end{array}$ & 1 January 2014 to 31 December 2018 \\
\hline $\begin{array}{l}\text { State National Hospital Cost Data Collection } \\
\text { (NHCDC) }\end{array}$ & $\begin{array}{l}\text { Records hospital costs, revenue and activity-based } \\
\text { funding data at the patient level for Queensland public } \\
\text { hospitals }\end{array}$ & 1 January 2014 to 31 December 2018 \\
\hline
\end{tabular}

A full list of variables to be requested from each data collection are listed in table 2.

*The Queensland Health Non-Admitted Patient Data Collection is only available for record linkage from 1 July 2016.

ICD-10, International Classification of Diseases-10th Revision. 


\section{Table 2 Data items to be requested}

\begin{tabular}{|c|c|}
\hline Data collection & Variables \\
\hline $\begin{array}{l}\text { Queensland Hospital } \\
\text { Admitted Patient Data } \\
\text { Collection }\end{array}$ & 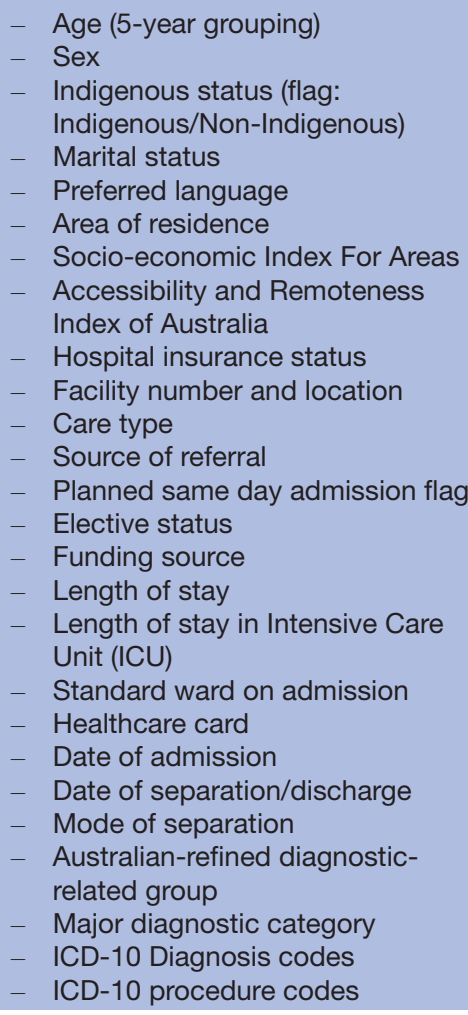 \\
\hline $\begin{array}{l}\text { Emergency Department } \\
\text { Information System }\end{array}$ & 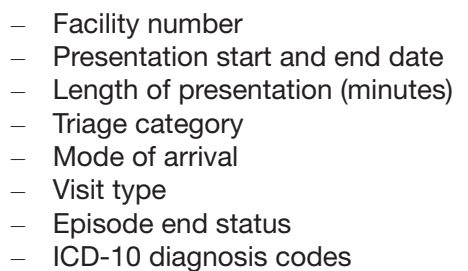 \\
\hline
\end{tabular}

$\begin{array}{ll}\text { Queensland Health Non- } & - \text { Date of service } \\ \text { Admitted Patient Data } & - \text { Service provider type } \\ \text { Collection } & - \text { Service delivery mode } \\ & - \text { Care type } \\ & - \text { Tier two clinic code } \\ \text { State National Hospital } & - \text { Direct cost per admission } \\ \text { Cost Data Collection } & - \text { Overhead cost per admission } \\ & - \text { Total cost per admission } \\ & - \text { Direct cost per ED presentation } \\ & - \text { Overhead cost per ED presentation } \\ \text { Registrar General deaths } & - \text { Total cost per ED presentation } \\ \text { Coded Cause of Death } & - \text { ICD coded cause of death } \\ \text { Data (Australian Bureau of } & \\ \text { Statistics) } & \end{array}$

ED, emergency department; ICD, International Classification of Diseases.

The level of health service use of people with epilepsy in Queensland, and costs associated with this, will be quantified using descriptive statistics including means, $\mathrm{SD}$ and IQRs. These estimates will be disaggregated to the type of health service use (hospital admission, ED presentation, outpatient appointment) as well as to population subgroups of interest including age, sex, Aboriginal and Torres Strait Islander status, rural/remote populations, and income status (stratified by those without and without low-income healthcare cards). Estimates will then be extrapolated to the Australian population using global burden of disease data on epilepsy prevalence by age and sex. All costs will be reported using annual estimates over the period of interest.

Factors associated with health service costs will be analysed using a Gamma regression model with explanatory variables including age, sex, indigenous status, rural/ remote status, low-income status as well as relevant clinical confounders including comorbidities. As individual income information will not be directly available, proxy measures for low-income status will be adopted including: whether a patient has access to a healthcare card (a concession card made available to individuals receiving specific government income support payments); a patient's health insurance status; and the Socio-Economic Index for Areas variable which describes the relative level of disadvantage of the area where an individual resides.

The average costs per patient will be quantified by subpopulation group and divided into quintiles. Logistic regression will be used to determine the odds of being a 'high cost patient' (highest cost quintile) in relation the various explanatory variables including age, sex, indigenous status, rural/remote status, low income status.

A comparative analysis will adopt two comparison groups with the aim of estimating the additional health service use and costs of epilepsy relative to other cohorts. The first comparison group will be a disease related cohort of patients admitted with any diagnosis of a nervous system disease. The second comparison group will include admissions randomly matched to the epilepsy sample by age, sex, indigenous status and remoteness of location. Linear, gamma and Poisson regression models will be used to estimate differences between epilepsy and comparison groups.

All costing data will be adjusted to reflect the year of publication using the Australian Consumer Price Inflation index, produced by the Australian Bureau of Statistics. ${ }^{19}$

\section{Limitations}

The use of hospital admissions data to identify the cohort of epilepsy patients will mean that not all patients are included. Specifically, healthy patients who have been successfully managing their condition in the community without being admitted to hospital are unlikely to be included in our sample. Patients with limited access to health services may also be excluded. Additional limitations of using administrative data are that accuracy of diagnostic data cannot be verified and that detailed clinical information and nuance is unavailable. Record linkage is often not $100 \%$ accurate, and there is a chance that not all individuals within the base dataset will be able to be linked across the other datasets, even where a true link exists. Record linkage also has the potential to make false-positive links. 


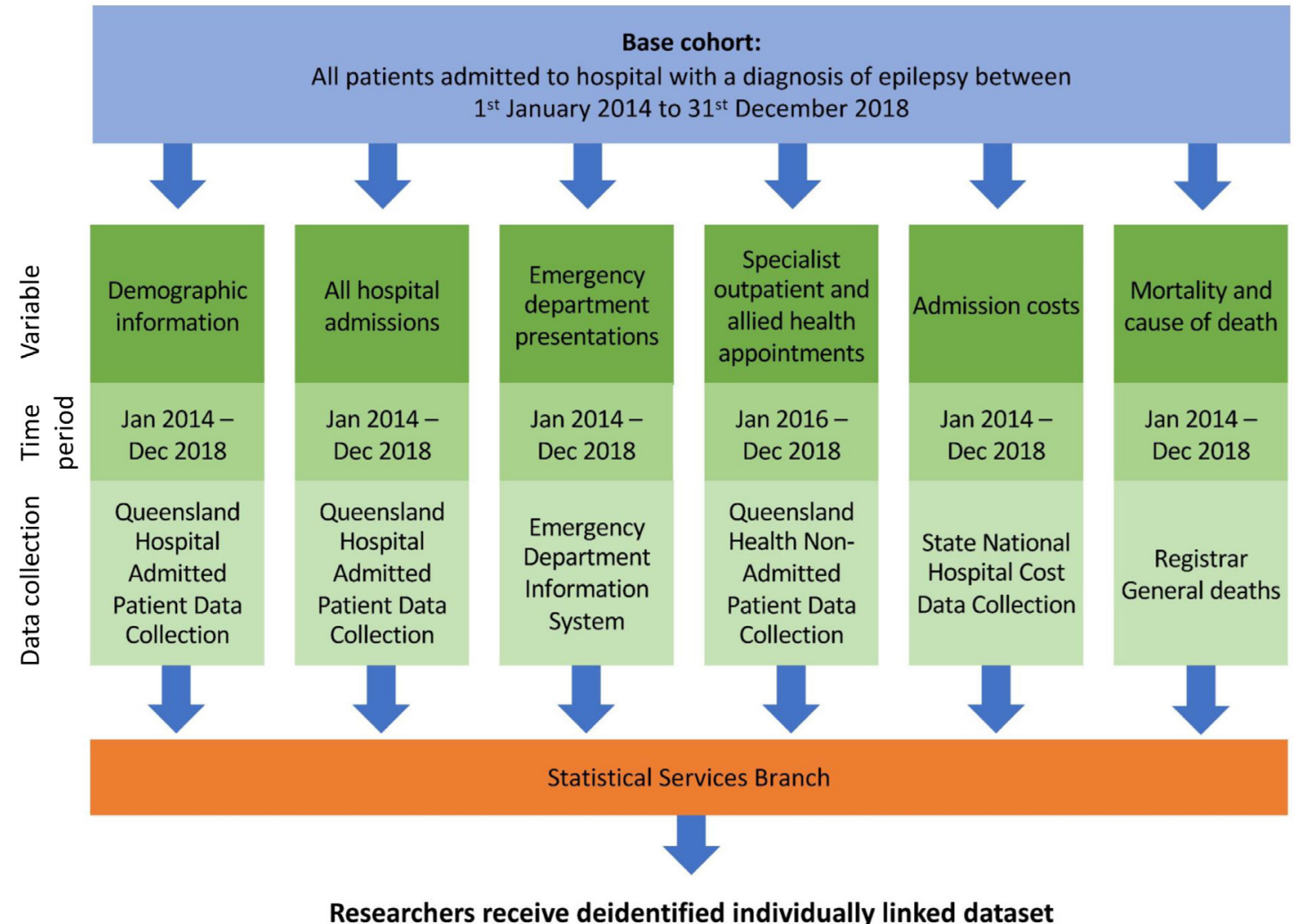

Figure 1 Data linkage process.

Within Australia, hospital care is the responsibility of state governments while primary care and pharmaceutical access are the responsibility of the national government. This has resulted in separate administrative and funding mechanisms, and fragmented datasets that are not readily available to be linked. Our dataset, therefore, does not include costs of primary care or pharmaceutical costs that are funded under national government schemes. Our analysis will, therefore, not be able to comprehensively quantify the health service use and cost of epilepsy in absolute terms. Rather, it will focus on the hospital setting and on the patients whose epilepsy is not well controlled, and in turn consuming a relatively large proportion of health system resources. Future studies are required to better understand the costs of epilepsy within primary and community care settings.

\section{ETHICS AND DISSEMINATION}

\section{Ethics approvals}

Low risk ethical approval has been obtained through the QUT University Human Research Ethics Committee (UHREC) (1900000333). Permission to waive consent has been sought and granted from Queensland Health under the Public Health Act 2005, with small cell counts to be supressed so that no identifiable (or potentially reidentifiable) patient information will be provided to the researchers. Signatures from all relevant data custodians on the PHA application have be obtained, with the final application submitted to the Health Innovation,
Investment and Research Office (HIIRO) for the Director General's final approval prior to the linkage process commencing.

\section{Data management}

Approved data provided by data custodians will be sent securely to researchers, and then held on secure electronic servers at QUT, available only to approved researchers within the study. Any hardware provided by data custodians (for example, datasets provided on compact disks (CDs)) will be kept in locked filing cabinets in a secure building at the university, available only to approved researchers within the study. All data will be managed in accordance with the QUT retention of records policy, with deidentified information collected on participants to be retained in a database in secure QUT folder, or stored in a locked cabinet, for 5 years following the last publication. After the required retention period has lapsed, electronic records will be deleted from the secure server, and any associate hardware (USBs, CDs, etc) will be wiped.

\section{Dissemination}

Findings of the proposed research will be communicated through presentations at national and international conferences, presentations to key stakeholders and decision-makers, and publications in international peerreviewed journals

Contributors HC, RT and SD conceived of and designed the study. HC and RB developed the data analysis plan. HC and RT led the principal drafting of the 
protocol, appraised the protocol, and managed drafting of revisions. All authors read and approved the final manuscript.

Funding This work was supported by an Institute of Health and Biomedical Innovation Early Career Researcher Development grant (IHBI ECR/MCR 2019 HC), through the Queensland University of Technology.

Competing interests None declared.

Patient and public involvement Patients and the public were not involved in the research question, design, conduct, or choice of outcome measures for the study. Australian and Queensland epilepsy advocacy bodies are assisting authors in engaging with the public and patients during dissemination activities, and to determine ongoing research activities arising from the results of data analysis.

Patient consent for publication Not applicable.

Provenance and peer review Not commissioned; externally peer reviewed.

Open access This is an open access article distributed in accordance with the Creative Commons Attribution Non Commercial (CC BY-NC 4.0) license, which permits others to distribute, remix, adapt, build upon this work non-commercially, and license their derivative works on different terms, provided the original work is properly cited, appropriate credit is given, any changes made indicated, and the use is non-commercial. See: http://creativecommons.org/licenses/by-nc/4.0/.

\section{ORCID iDs}

Ruth Tulleners http://orcid.org/0000-0002-9678-6436

Robin Blythe http://orcid.org/0000-0002-3643-4332

Hannah Carter http://orcid.org/0000-0002-0046-4126

\section{REFERENCES}

1 Moshé SL, Perucca E, Ryvlin P, et al. Epilepsy: new advances. Lancet 2015:385:884-98

2 Vos T, Barber RM, Bell B, et al. Global, regional, and national incidence, prevalence, and years lived with disability for 301 acute and chronic diseases and injuries in 188 countries, 1990-2013: a systematic analysis for the global burden of disease study 2013. Lancet 2015;386:743-800.

3 Murray CJ, Lopez AD. Global comparative assessments in the health sector: disease burden expenditures and intervention packages, 1994.

4 Loney JC, Wirrell EC, Sherman EMS, et al. Anxiety and depressive symptoms in children presenting with a first seizure. Pediatr Neurol 2008;39:236-40.
5 Fuller-Thomson E, Brennenstuhl S. The association between depression and epilepsy in a nationally representative sample. Epilepsia 2009;50:1051-8.

6 Australian Bureau of Statistics. National health survey 2004-05: Smmary of results cat. No. 4364.0. Canberra, 2006.

7 Bellon ML, Barton C, McCaffrey N, et al. Seizure-Related hospital admissions, readmissions and costs: comparisons with asthma and diabetes in South Australia. Seizure 2017;50:73-9.

8 Strzelczyk A, Reese JP, Dodel R, et al. Cost of epilepsy. Pharmacoeconomics 2008;26:463-76.

9 Berg AT. Understanding the delay before epilepsy surgery: who develops intractable focal epilepsy and when? CNS Spectr 2004;9:136-44.

10 Jetté N, Quan H, Faris P, et al. Health resource use in epilepsy: significant disparities by age, gender, and Aboriginal status. Epilepsia 2008;49:586-93.

11 Vaughan KA, Lopez Ramos C, Buch VP, et al. An estimation of global volume of surgically treatable epilepsy based on a systematic review and meta-analysis of epilepsy. J Neurosurg 2018;130:1127-41.

12 Solli E, Colwell NA, Say I, et al. Deciphering the surgical treatment gap for drug-resistant epilepsy (DRE): a literature review. Epilepsia 2020;61:1352-64.

13 Begley CE, Famulari M, Annegers JF, et al. The cost of epilepsy in the United States: an estimate from population-based clinical and survey data. Epilepsia 2000;41:342-51.

14 Mitchell RJ, Herkes G, Nikpour A, et al. Examining health service utilization, hospital treatment cost, and mortality of individuals with epilepsy and status epilepticus in New South Wales, Australia 20122016. Epilepsy Behav 2018;79:9-16.

15 Australian Bureau of Statistics. Regional population growth, Australia, 2018-19: population estimates by remoteness area, 2009 to 2019, 2020. Available: https://www.abs.gov.au/AUSSTATS/abs@. nsf/DetailsPage/3218.02018-19?OpenDocument [Accessed 08 Jun 2020].

16 Davy C, Cass A, Brady J, et al. Facilitating engagement through strong relationships between primary healthcare and Aboriginal and Torres Strait Islander peoples. Aust N Z J Public Health 2016;40:535-41.

17 Thurman DJ, Beghi E, Begley CE, et al. Standards for epidemiologic studies and surveillance of epilepsy. Epilepsia 2011;52 Suppl 7:2-26.

18 Tan M, Wilson I, Braganza V, et al. Development and validation of an epidemiologic case definition of epilepsy for use with routinely collected Australian health data. Epilepsy Behav 2015;51:65-72.

19 Australian Bureau of Statistics. Consumer price index, Australia. Canberra: Commonwealth of Australia, 2020. 